\title{
CONVERGENCE OF THE AVERAGES AND FINITENESS OF ERGODIC POWER FUNCTIONS IN WEIGHTED $L^{1}$ SPACES
}

\author{
Pedro Ortega Salvador
}

\begin{abstract}
Let $(X, \mathcal{F}, \mu)$ be a finite measure space. Let $T: X \rightarrow X$ be a measure preserving transformation and let $A_{n} f$ denote the average of $T^{k} f, k=$ $0, \ldots, n$. Given a real positive function $v$ on $X$, we prove that $\left\{A_{n} f\right\}$ converges in the a.e. sense for every $f$ in $L^{1}(v d \mu)$ if and only if inf $i \geq 0 v\left(T^{i} x\right)>$ 0 a.e., and that the same condition is equivalent to the finiteness of a related ergodic power function $P_{r} f$ for every $f$ in $L^{1}(v d \mu)$. We apply this result to characterize, being $T$ null-preserving, the finite measures $\nu$ for which the sequence $\left\{A_{n} f\right\}$ converges a.e. for every $f \in L^{1}(d \nu)$ and to prove that uniform boundedness of the averages in $L^{1}$ is sufficient for finiteness a.e. of $P_{\mathrm{r}}$.
\end{abstract}

\section{Introduction}

Let $(X, \mathcal{F}, \mu)$ be a finite measure space and let $T: X \longrightarrow X$ be a measure preserving transformation. For every measurable function $f$ on $X$ we consider the averages

$$
A_{n} f=(n+1)^{-1} \sum_{j=0}^{n} T^{j} f
$$

where $T^{j} f(x)=f\left(T^{j} x\right)$, the maximal operator

$$
M f=\sup _{n \geq 0} A_{n}|f|
$$

and the power function

$$
P_{r} f=\left(\sum_{n=0}^{\infty}\left|A_{n+1} f-A_{n} f\right|^{r}\right)^{1 / r} \quad(r>1) .
$$

This research has been supported by C.A.I.C.Y.T. Grant (PB85-0434). 
In [7], Martin Reyes and A. de la Torre characterized the positive measurable functions $v$ such that $\left\{A_{n} f\right\}$ converges a.e. for all $f$ in $L^{1}(v d \mu)$ as those functions that verify

$$
\inf _{i \geq 0} v\left(T^{i} x\right)>0 \text { a.e. }
$$

(see also [15] for a ratio theorem).

Section 3 of this note is devoted to give a simpler proof for this result and to prove a similar theorem for $P_{r}$. It is seen that condition (1.1) is also valid for $P_{r}$. The main tools we use are Nikishin's theorem and conditional expectations which solve the problems derived from the non-invertibility of the transformation. These technics have been recently used to solve the problem of the convergence of the averages for $p>1$. (see [8]).

As a previous result, we have to state the weak type $(1, I)$ for $P_{r}$. This question was solved in [17] by Yoshimoto. Our approach is different, but suitable for our purposes. It was also treated in [5] and [11], but under more restrictive conditions.

Finally, in section 4 we work with a null-preserving transformation $T$ and characterize the finite measures $\nu$ for which the sequence of the ergodic averages $\left\{A_{n} f\right\}$ converges a.e. for every $f \in L^{1}(d \nu)$ as those measures with the property: there exists a measure $\gamma$ equivalent to $\nu$ such that

$$
\gamma(\{x \in X / M f(x)>\lambda\}) \leq \lambda^{-1} \int_{X}|f| d \nu
$$

for every $f \in L^{1}(d \nu)$.

In [13], Ryll-Nardzewski characterized the finite measures $\nu$ for which the ergodic averages $\left\{A_{n} f\right\}$ converge a.e. to a $L^{1}$-function for every $f \in L^{1}(d \nu)$ as those measures that verify Hartman's condition: there exists a constant $K$ such that

$$
\lim \sup _{n} n^{-1} \sum_{i=0}^{n-1} \nu\left(T^{-i} E\right) \leq K \nu(E)
$$

for every set $E$.

Our result is different from the Ryll-Nardzevsky's one, because we allow the limit function not to be in $L^{1}(d \nu)$. This situation is possible as Dowker's example shows (see [1] and, for a two-dimensional version, see [12]) and, therefore, our condition is strictly weaker than Hartman's condition.

As a corollary, we prove that uniform boundedness of the avcrages is a sufficient condition for finiteness of $P_{r}$ for every $f \in L^{1}$. This result is a $L^{1}$ version of theorem 3.1 in [10]. Other references about $P_{r}$ are [14] and [16]. 


\section{Previous results}

We will need two lemmas and scveral results about the operators $P_{r}, q_{r}$ and $Q_{r}$, where $q_{r}$ is defined on functions on $\mathbf{N}$, the set of the natural numbers, by

$$
q_{r} a(i)=\left(\sum_{k=0}^{\infty}|a(i+k)|^{r}(k+1)^{-r}\right)^{1 / r} \quad(i \in N)
$$

and $Q_{r}$ on functions on $X$ by

$$
Q_{r} f(x)=\left(\sum_{k=0}^{\infty}\left|f\left(T^{k} x\right)\right|^{r}(k+1)^{-r}\right)^{1 / r}
$$

Lemma 1. Let $k$ be a natural number. Then, there exists a countable family $\left\{B_{i}: i \in \mathbf{N}\right\}$ of measurable sets such that

i) $X=\dot{U}_{i} B_{i}$

ii) $B_{i} \cap B_{j}=\phi$ if $i \neq j$

iii) For every $i$, there exisis a natural number $s(i)$ with $0 \leq s(i) \leq k$ such that the sets $\left\{T^{-j} B_{i}: 0 \leq j \leq s(i)\right\}$ are pairwise disjoint and such that if $s(i)<k$ then $T^{-1-s(i)} A=A$ for every subset $A$ of $B_{i}$. Consequently, for every subset $A$ of $B_{i}$

$$
\sum_{j=0}^{k} \chi_{T^{-j} A} \leq C(i) \sum_{j=0}^{s(i)} \chi_{T^{-j} A} \leq 2 \sum_{j=0}^{k} \chi_{T^{-j} A}
$$

where $C(i)$ is the least integer bigger than or equal to $(k+1)(1+s(i))^{-1}$.

For the proof see lemma (2.10) in [9] changing $T^{h}$ by $T^{-h}$.

Lemma 2. Let $(X, \mathcal{F}, \mu)$ be a finite measure space ond let $\left\{\mathcal{F}_{n}\right\}$ be a decreasing sequence of sub- $\sigma$-algebras. Let $\mathcal{F}_{\infty}=\cap_{n} \mathcal{F}_{n}$ and denote by $E_{n}$ the conditional expectation with respect to $\mathcal{F}_{n}$. If $\left\{f_{n}\right\}$ is an a.e. convergent sequence of functions such that $\left|f_{n}\right| \leq C$ a.e. and $f$ is the a.e. limit of $f_{n}$ then $E_{\infty} f$ is the a.e. limit of $E_{n} f_{n}$.

This lemma follows from theorem 7.6 in $[6]$ and the decreasing martingale theorem.

Theorem 1. $q_{r}$ is of weak type $(1,1)$ with respect to the counting measure on $\mathrm{N}$.

Proof: The proof is the same as the one of theorem (3.8) in [10] with obvious changes derived from the facts that we are working in $N$ and that lemma (3.2) (in [10]) is not necessary. 
Theorem 2. $Q_{r}$ is of weok type $(1,1)$.

Proof: It follows from theorem 1 and transference arguments (see [11]).

Theorem 3. $P_{r}$ is of weak type $(1,1)$ and, as a consequence, the series

$$
\sum_{k=0}^{\infty}\left|A_{k+1} f-A_{k} f\right|^{r}
$$

is a.e. convergent for every $f$ in $L^{1}(d \mu)$.

Proof: It follows inmediately by theorem 2, the ergodic theorem and the well-known inequality

$$
P_{r} f \leq C M f+Q_{r} f
$$

Remark. Note that theorems 2 and 3 do not need finiteness of the measure space.

\section{Main result}

Theorem 4. Let $(X, \mathcal{F}, \mu)$ be a finite measure space. Let $T: X \longrightarrow X$ be a measure preserving transformation. Let $v$ be a positive measurable function on $X$. The following are equivalent:
a) The sequence $\left\{A_{n} f\right\}$ converges a.e. for all $f$ in $L^{1}(v d \mu)$.
b) $\sum_{k=0}^{\infty}\left|A_{k+1} f-A_{k} f\right|^{r}<\infty$ in the a.e. sense for all $f$ in $L^{1}(v d \mu)$.
c) $\sum_{k=0}^{\infty}(k+1)^{-r}\left|T^{k} f\right|^{r}<\infty$ in the a.e. sense for all $f$ in $L^{1}(v d \mu)$.
d) $M f<\infty$ a.e. for all $f$ in $L^{1}(v d \mu)$.
e) There exists a positive measurable function $u$ such that $\int_{\{x: M f(x)>\lambda\}} u d \mu$ $\leq \lambda^{-1} \int_{X}|f| v d \mu$ for all $\lambda>0$ and all $f$ in $L^{1}(v d \mu)$.
f) There exists a positive measurable function $u$ such that $\sup _{k \geq 0}$ $\int_{\left\{x: A_{k} f(x)>\lambda\right\}} u d \mu \leq \lambda^{-1} f_{X}|f| v d \mu$ for all $\lambda>0$ and all $f$ in $L^{2}(v d \mu)$.
g) There exists a positive measurable function $u$ such that $\int_{\left\{x: P_{+} f(x)>\lambda\right\}} u d \mu$ $\leq \lambda^{-1} \int_{X}|f| v d \mu$ for all $\lambda>0$ and all $f$ in $L^{1}(v d \mu)$.
h) There exists a positive measurable function $u$ such that $\int_{\left\{x: Q_{r} f(x)>\lambda\right\}} u d \mu$ $\leq \lambda^{-1} \int_{X}|f| v d \mu$ for all $\lambda>0$ and all $f$ in $L^{1}(v d \mu)$.
i) $\inf _{i \geq 0} v\left(T^{i} x\right)>0$ a.e.

Proof: Implications a) $\Rightarrow$ d) and e) $\Rightarrow f$ ) are clear. d) implies e), b) implies g) and c) implies h) by Nikishin's theorem (see [2] pages 536-537 and [3]). Nikishin's theorem needs the continuity in measure of the operators $M, P_{r}$ and $Q_{r}$ from $L^{1}(v d \mu)$ to $L^{0}(d \mu)$. This condition follows by theorem 1.1.1 in [4], page 10. 
f) $\Rightarrow$ i) We may assume $u \leq 1$. Let $k$ be a nonnegative integer. Let $\left\{B_{i}\right\}$ be the sequence of sets associated to $k$ by lemma 1 . Fix $i$ and let $A$ be a measurable subset of $B_{i}$. Let $R=\cup_{0 \leq j \leq k} T^{-j} A=\cup_{0 \leq j \leq s(i)} T^{-j} A$. It is clear that $R$ is contained in $\left\{x: A_{k}\left(\chi_{A}\right)(x) \geq C(i)(k+1)^{-1}\right\}$. Then $\left.\mathrm{f}\right)$, lemma 1 and the fact that $T$ is m.p.t. give

$$
\begin{array}{r}
\int_{T^{-k} A} \sum_{j=0}^{k} u\left(T^{j} x\right) d \mu=\sum_{j=0}^{k} \int_{T^{-j} A} u d \mu \leq C(i) \sum_{j=0}^{s(i)} \int_{T^{-j} A} u d \mu=C(i) \int_{R} u d \mu \\
\leq(k+1) \int_{A} v d \mu=(k+1) \int_{T^{-k} A} v\left(T^{k} x\right) d \mu .
\end{array}
$$

The above inequality has been proved for a measurable subset $A$ of $B_{i}$. Since $X=\cup_{i} B_{i}$, it is clear that the inequality is true for every measurable subset $A$ of $X$ and therefore if $E_{k}$ is the conditional expectation with respect to the sub- $\sigma$-algebra $T^{-k} \mathcal{F}$ we have

$$
E_{k}\left((k+1)^{-1} \sum_{j=0}^{k} T^{j} u\right)(x) \leq T^{k} v(x) \text { a.e. } x \in X .
$$

Taking lim inf when $k$ tends to infinity, Birkhoff's theorem and lemma 2 give

$$
E u(x) \leq \lim \inf _{k \rightarrow \infty} T^{k} v(x) \text { a.e. } x \in X,
$$

where $E u$ is the conditional expectation of $u$ with respect to the sub- $\sigma$-algebra of the invariant sets.

Since $E u$ is positive a.e., we obtain $\inf _{k \geq 0} v\left(T^{k} x\right)>0$ a.e. .

g) $\Rightarrow$ i) We may assume $u \leq 1$. Let $k$ be a natural number and $\left\{B_{i}\right\}$ be the sequence of sets given by lemma 1. Fix $i$ with $s(i)>0$ and let $A$ be contained in $B_{i}$. Let $R=\cup_{0 \leq j \leq k} T^{-j} A=\cup_{0 \leq j \leq s(i)} T^{-j} A$. Let's see that $R-A$ is contained in $\left\{x: P_{r}\left(\chi_{A}\right)(x) \geq(1+s(i))^{-1}\right\}$.

Let $y \in R-A$. There exists one and only one $h$ with $0<h \leq s(i)$ such that $T^{h} y \in A$. Then

$$
P_{r}\left(\chi_{A}\right)(y) \geq\left|(h+1)^{-1} \sum_{j=0}^{h} \chi_{A}\left(T^{j} y\right)-h^{-1} \sum_{j=0}^{h-1} \chi_{A}\left(T^{j} y\right)\right| \geq(1+s(i))^{-1}
$$

Therefore g) gives

$$
\int_{R-A} u d \mu \leq(1+s(i)) \int_{A} v d \mu
$$


Since $u \leq v$ we have

$$
\int_{R} u d \mu \leq(2+s(i)) \int_{A} v d \mu
$$

Recall that we have been working with $s(i)>0$. But if $s(i)=0$ the last inequality is trivial. Then

$$
\int_{T-k_{A}} \sum_{j=0}^{k} u\left(T^{j} x\right) d \mu \leq C(i) \int_{R} u d \mu \leq C(i)(s(i)+2) \int_{A} v d \mu \leq 4(k+1) \int_{A} v d \mu .
$$

Now, the same argument used in the above implication gives i).

h) $\Rightarrow$ i) Let $k,\left\{B_{i}\right\}, A$ and $R$ as in $\left.\left.\mathrm{f}\right) \Rightarrow \mathrm{i}\right)$. It is easy to see that $R$ is contained in $\left\{x: Q_{r}\left(\chi_{A}\right)(x)>(1+s(i))^{-1}\right\}$. Then, the argument follows as in f) $\Rightarrow$ i).

i) $\Rightarrow$ a) The proof of this implication can be seen in [7]. We include it for this section to be selfcontained.

Let $B_{k}=\left\{x: \inf _{i \geq 0} v\left(T^{i} x\right)<2^{-k}\right\} . B_{k}$ and $X-B_{k}$ are invariant under $T$ and since $v(x) \geq 2^{-\bar{k}}$ on $X-B_{k}$ we have that $L^{1}\left(X-B_{k}, v d \mu\right)$ is contained in $L^{1}\left(X-B_{k}, d \mu\right)$. Then Birkhoff's theorem shows that $\left\{A_{n} f\right\}$ converges a.e. on $X-B_{k}$ for every $f \in L^{1}\left(X-B_{k}, v d \mu\right)$. Since $\lim _{k} \mu\left(B_{k}\right)=0$ by (i), we obtain (a).

Finally, i) $\Rightarrow$ b) and i $\Rightarrow$ c) by the same argument that the above but using theorems 3 and 2 respectively in place of Birkhoff's theorem.

\section{Convergence of the averages and finiteness of $P_{r}$ in the general case}

Theorem 5. Let $(X, \mathcal{F}, \nu)$ be a finite measure space and let $T: X \longrightarrow X$ be a null-preserving transformation. The following statements are equivalent:

a) There exists a measure $\gamma$ equivalent to $\nu$ such that

$$
\gamma(\{x \in X / M f(x)>\lambda\}) \leq \lambda^{-1} \int_{X}|f| d \nu
$$

for every $f \in L^{1}(d \nu)$.

b) There exists a measure $\gamma$ equivalent to $\nu$ such that

$$
\sup _{n \geq 0} \gamma\left(\left\{x \in X / A_{n}|f|(x)>\lambda\right\}\right) \leq \lambda^{-1} \int_{X}|f| d \nu
$$

for every $f \in L^{1}(d \nu)$.

c) $\left\{A_{n} f\right\}$ converges a.e. for every $f \in L^{1}(d \nu)$. 
d) $M f(x)<\infty$ a.e. for every $f \in L^{1}(d \nu)$.

Moreover, if one of the above conditions holds, then $Q_{r} f$ and $P_{r} f$ are finite a.e. for every $f \in L^{1}(d \nu)$.

Proof: Implications a) $\Rightarrow$ b) and c) $\Rightarrow d$ ) are obvious. On the other hand, a) follows from d) by Nikishin's theorem. We only have to prove b) $\Rightarrow c$ ). Simultaneously, we will see that b) implies finiteness a.e. for $Q_{r}$ and $P_{r}$.

From b) and Marcinkiewicz's interpolation theorem we have

$$
\sup _{k \geq 0} \int_{X}\left|A_{k} f\right|^{2} d \gamma \leq C \int_{X}|f|^{2} d \nu \text { for every } f \in L^{2}(d \nu) .
$$

Let $L$ be a Banach's limit (for instance see $[6]$ ) and define

$$
\mu(E)=L\left(\left\{\int_{X} A_{k} \chi_{E} d \gamma\right\}\right) \quad(E \in \mathcal{F}) .
$$

$\mu$ is well defined by (4.1). $\mu$ is an invariant measure and it is absolutely continuous with respect to $\nu$. Let $v$ be the Radon-Nikodym derivative $d \mu / d \nu, D=$ $\{x: v(x) \neq 0\}$ and $Y=\cap_{n \geq 0} T^{-n} D$. It is clear that $\mu(X-Y)=0$ and $\left.T\right|_{Y}$ applies $Y$ in $Y$. Therefore we have that $\left.\nu\right|_{Y}$ is equivalent to the invariant measure $\left.\mu\right|_{Y}$. Then it follows by theorem 4 that the averages $\left\{A_{k} f\right\}$ converge and $M f, Q_{r} f$ and $P_{r} f$ are finite a.e. $(\nu)$ in $Y$ for every $f \in L^{1}(d \nu)$.

To prove the a.e. $(\nu)$ convergence of $\left\{A_{k} f\right\}$ and the finiteness of $M f, Q_{r} f$ and $P_{r} f$ on $X-Y$ we shall first statc that for almost all $x(\nu)$ in $X$ there exists $n$ such that $T^{n} x \in Y$. If this property is not true, then there exists $B$ with $\nu(B)>0$ such that for every $i, B$ is containcd in $T^{-i}(X-Y)$. Then for every $k$

$$
\gamma(B) \leq(k+1)^{-1} \sum_{i=0}^{k} \gamma\left(T^{-i}(X-Y)\right)=\int_{X} A_{k} X X-Y d \gamma
$$

and the properties of Banach's limits give

$$
\gamma(B) \leq L\left(\left\{\int_{X} A_{n} \chi X-Y d \gamma\right\}\right)=\mu(X-Y)=0,
$$

which goes against $\nu(B)>0$ since $\gamma$ and $\nu$ are equivalent.

Let $x$ be in $X-Y$ and let $n$ be an integer verifying $T^{n} x \in Y$. Let $k \geq n$. Then

$$
\begin{gathered}
A_{k} f(x)=(k+1)^{-1}\left(\sum_{i=0}^{n-1} f\left(T^{i} x\right)\right)+(k-n+1)(k+1)^{-1}(k-n+1)^{-1} \sum_{i=n}^{k} f\left(T^{i} x\right) \\
\begin{array}{r}
\text { and } \sum_{j=0}^{k}(j+1)^{-r}\left|f\left(T^{j} x\right)\right|^{r} \leq \sum_{j=0}^{n-1}(j+1)^{-r}\left|f\left(T^{j} x\right)\right|^{r} \\
\quad+\sum_{j=\Lambda}^{k}(j-n+1)^{-r}\left|f\left(T^{j} x\right)\right|^{r}
\end{array}
\end{gathered}
$$


Since $T^{n} x \in Y$ and $T$ applies $Y$ in $Y$, when $k$ tends to infinity we obtain finite limits. Therefore, we have proved that $\left\{A_{k} f(x)\right\}$ converges a.e. and that $M f(x)$ and $Q_{r} f(x)$ are finite a.e. for every $f$ in $L^{1}(d \nu)$. Then, since $P_{r} f \leq C M f+Q_{r} f$ we obtain the finiteness of $P_{r}$.

Corollary. Let $(X, \mathcal{F}, \nu)$ be a finite measure space and let $T: X \longrightarrow X$ be a null-preserving transformation. If $\sup _{k \geq 0}\left\|A_{k}\right\|_{1}<\infty$ then $\left\{A_{k} f\right\}$ converges a.e. and $M f, Q_{r} f$ and $P_{r} f$ are finite o.e. for every $f \in L^{1}(d \nu)$.

Acknowledgements. I have to thank Professor Martín Reyes for his continuous help. Also, I would like to thank the referee for his helpful comments and suggestions.

\section{References}

1. YaEl NaIM DOWKer, A note on the ergodic theorems, Bull. Amer. Math. Soc. 55 (1949), 379-383.

2. J. García CUerVa AND J.L. Rubio de Francla, "Weigthed notm inequalities and related topics," North Holland, 1985.

3. J.E. GiLBERT, Nikishin-Stein theory and factorization with applications, Proc. Sympos. Pure Math. 35, 2 (1979).

4. M. DE GUZMÁN, "Real variable methods in Fourier analysis," North Holland, $198 \mathrm{I}$.

5. R.L. JONES, Inequalities for the ergodic maximal function, Studia Math. $60(1977), 101-129$.

6. U. KRENGeL, "Ergodic theorems," Walter de Gruyter, 1985.

7. F.J. MARTíN-REYes AND A. DE LA TORRE, Weigthed weak type inequalities for the ergodic maximal function and the pointwise ergodic theorem, Studia Math. 87 (1987), 33-46.

8. F.J. MARTÍn-REyes AND A. DE LA TORRE, On the almost everywhere convergence of the ergodic averages, Ergod. Th. and Dinam. Sys. 10 (1990), $141-149$.

9. F.J. MaRTín-ReYes, Inequalities for the maximal function and convergence of the averages in weighted $L^{p}$-spaces, Trans. Amer. Math. Soc. 296 (1986), 61-82.

10. F.J. Martín-Reyes and P. Ortega Salvador, Ergodic power functions for mean bounded, invertible, positive operators, Studia Math. 91, 2 (1988), 131-139.

11. F.J. MARTín-ReYeS, Weights for ergodic square functions, Ann. Inst. Henri Poincare (Probabilites et Statisques) 22, 3 (1986), 333-345. 
12. F.J. MARTíN-REYeS, On a pointwise ergodic theorem for multiparameter groups, Proceedings of the International convergence on almost everywhere convergence held in Columbus, Ohio (1988), 267-279.

13. C. RYLL-NARDzEWSKI, On the ergodic theorems (I) (Generalized ergodic theorems), Studia Math. 12 (1951), 65-73.

14. R. SATO, On the crgodic power function for invertible positive operators, Studia Math. 90, 2 (1988), 129-134.

15. R. Sato, On a ratio ergodic theorem, Studia Math. 87 (1987), 47-52.

16. A. DE LA TORRE, The square function for $L^{1}-L^{\infty}$ contractions, Proc. Sympos. Pure Math. 35, 2 (1979), 435-438.

17. T. Yoshimoro, Some inequalities for ergodic power functions, Act. Math. Acad. Sci. Hung. 36 (1-2) (1980), 19-24.

\author{
Deparlamento de Estadística y Econometría \\ Facultad de Económicas \\ Universidad de Málaga \\ 29013 - Málaga \\ SPAIN
}

Primera versió rebuda el 20 de Febrer de 1990 , darrera versió rebuda el 3 d'Agost de 1990 